\title{
Reviewers for The Journal of the American Board of Family Practice in 2004
}

Peer reviewers are critical to the success and quality of the $\mathcal{A} A B F P$. Here we acknowledge and thank the many individuals who, during 2004, volunteered their time and expertise to read and critique submissions for the journal. Peer reviewing is a labor of love. Top researchers in primary care and other specialties provide thoughtful recommendations and insights to their colleagues. This devotion to advancing primary care research and practice, plus the commitment to nurturing their colleagues is a tremendous service to us all. If you submitted a peer review to $7 A B F P$ in 2004 and your name is not listed, please let us know. Readers are invited to enlist as peer reviewers for the $7 A B F P$. We want to expand our pool of reviewer expertise, and the scholarly perspectives in the critiques and suggestions that we provide to authors. You can become a $7 A B F P$ peer reviewer by downloading the registration form from http://www.jabfp.org. Click the "Peer Reviewers" button on the left column of the home page to access the form.

Andy Abril

Louise Acheson

David Acosta

Ameena T. Ahmed

William Alto

M. R. Anderson

R. Anton

Macaran A. Baird

J. Banks-Wallace

Steven L. Barnett

Barnette (first name unknown)

S. Basaria

Kay A. Bauman

Elizabeth Bayliss

Pamela Bean

Ettore Beghi

Franco Benazzi

Ian M. Bennett

Vance Berger

James Bergman

Arlene Bierman

James R. Blackman

Mary Blehar

Carol E. Blenning
Amy Bode

Hillary Bogner

Rachel Bramson

Kent Bream

Brucker (first name unknown)

Sean T. Bryan

Stephen A. Buetow

Sandra K. Burge

Doug Campos-Outcalt

Christina Chan

Frederick M. Chen

Loren Z. Chiu

J. E. Cleary-Goldman

Marsha M. Cohen

Jack M. Colwill

David D. Cravens

Renee M. Crichlow

Peter Cronholm

Michael A. Crouch

Brian Crownover

Patrick Culligan

Larry Culpepper

Timothy Daaleman

Alan K. David
Darwin Deen

Frank DeGruy

Mark J. DeHaven

Dianne Delva

Raymond Y. Demers

William W. Dexter

Lori M. Dickerson

Steven A. Dosh

David Doukas

Benjamin Druss

Christine Duffy

S. Shevaun Duiker

Scott Eathorne

Nancy C. Elder

John W. Ely

Michael E. Ernst

Stuart J. Farber

Kenneth S. Fink

Patrick Finley

Cheryl Flynn

Samuel N. Forjuoh

Arthur Fournier

Andrea S. Franks

Peter Franks 


\begin{tabular}{|c|c|c|}
\hline E. W. Freeman & Colin P. Kopes-Keer & Donald E. Nease Jr. \\
\hline John J. Frey III & Kent K. Krack & Richard Neill \\
\hline Keith A. Frey & N. R. Kressin & Warren P. Newton \\
\hline Sim S. Galazka & K. Laederach-Hofmann & Jim Nicholson \\
\hline Lillian Gelberg & Jennifer E. Lafata & Thomas E. Norris \\
\hline John Geyman & Landgraf (first name unknown) & Paul Nutting \\
\hline Nassir Ghaemi & Michael Lauer & A. N. Ortega \\
\hline Valerie J. Gilchrist & Michael LeFevre & Thomas Oxman \\
\hline James Gill & Bernard Lo & Anuradha Paranjape \\
\hline Mary Lou L. Gillard & C. Locke & Donald Pathman \\
\hline Dwenda K. Gjerdingen & Everett Logue & Katherine Patterson-Neely \\
\hline Marji Gold & Franco Lumachi & Randolph L. Pearson \\
\hline Adam O. Goldstein & P. J. Lustman & R. P. Petchey \\
\hline Bernard Gonik & D. Lynge & Lyle R. Petersen \\
\hline Ken Grauer & Charles D. MacLean & R. P. Pierzchajlo \\
\hline Judith A. Gravdal & Diane Jean Madlon-Kay & Linda W. Prine \\
\hline Jocelyn Gravlee & Michael K. Magill & Michael D. Prislin \\
\hline Beverly Green & Debbi Main & Howard K. Rabinowitz \\
\hline Larry Green & Arch G. Mainous III & Donald Ransom \\
\hline Anita R. Gross & R. J. Marchiando & Shobha S. Rao \\
\hline Wayne A. Hale & Kyriakos S. Markides & Lisa J. Rapport \\
\hline W. L. Hall II & Tsevti Markova & Robert Ratner \\
\hline Rebecca Hardy & Jeffrey Martin & E. S. Rawson \\
\hline Susan Hardy & I. Martinelli & Janet Realini \\
\hline J. Hayano & Samuel C. Matheny & Barbara D. Reed \\
\hline R. P. Heaney & Wasim Maziak & William Reichel \\
\hline Bernhard Hellmich & M. R. McGuigan & Michael D. Reis \\
\hline Eric Henley & Helen McIlvain & Rick E. Ricer \\
\hline E. Heron & Joel H. Merenstein & D. J. Rickert \\
\hline John M. Hicker & Aaron Michelfelder & K. J. Rinnert \\
\hline N. S. Howden & John E. Midtling & W. David Robinson \\
\hline William J. Hueston & James W. Mold & William MacMillan Rodney \\
\hline Carlos R. Jaen & M. H. Monroe & John C. Rogers \\
\hline Julie Jonassen & Hugh Mulcahy & Roger A. Rosenblatt \\
\hline Wayne Katon & John B. Murphy & Jo Ann Rosenfeld \\
\hline T. Jeffrey Kirchner & M. H. Murphy & Thomas A. Ruane \\
\hline Joseph C. Konen & Elza Muscelli & Mack T. Ruffin \\
\hline
\end{tabular}


Terry S. Ruhl

Sanjay Saint

Maryjean Schenk

Joseph E. Scherger

L. Schwartz

Thomas L. Schwenk

John Sear

James J. Sejvar

Sherer (first name unknown)

Paul Sloan

Philip D. Slone

Naomi Smidt-Afek

John Spangler

Stephan Spann

Jeffrey Staab
Howard F. Stein

Meir Steiner

G. Gayle Stephens

M. David Stockton

Joseph Straton

Barbara Supanich

Jeff L. Susman

Thomas R. Taylor

Peter G. Teichman

Frank Thien

Daniel J. Van Durme

C. J. Van Gool

Eric Wall

Barry Weiss

Myrna Weissman
Paul T. Werner

Patricia West

Robert A. Wild

Calvin L. Wilson

Stephen A. Wilson

K. L. Wisner

Robert M. Wolfe

Xiao $\mathrm{Wu}$

W. Yancy

Barbara P. Yawn

Richard A. Young

Atif Zafar

Atif Zaman

Gregory S. Zaric

Therese Zink 